\title{
LEONARD EUGENE DICKSON 1874-1954
}

Leonard Eugene Dickson was born in Independence, Iowa on January 22,1874 . He was a brilliant undergraduate at the University of Texas receiving his B.S. degree as valedictorian of his class in 1893. He was a chemist with the Texas Biological Survey from 1892-1893. He served as a teaching fellow at the University of Texas receiving the M.A. degree in 1894 . He held a fellowship at the University of Chicago from 1894 to 1896 and was awarded its first Ph.D. in Mathematics in 1896. He spent the year 1896-1897 in Leipzig and Paris, was instructor in mathematics at the University of California 1897-1899, Associate Professor at Texas 1899-1900, Assistant Professor at Chicago 1900-1907, Associate Professor 1907-1910, and Professor in 1910. He was appointed to the Eliakim Hastings Moore Distinguished Professorship in 1928, and became Professor Emeritus in 1939. He served as Visiting Professor at the University of California in 1914, 1918, and 1922.

Professor Dickson was awarded the $\$ 1,000$ A.A.A.S. Prize in 1924 for his work on the arithmetics of algebras. He was awarded the Cole Prize of the American Mathematical Society in 1928 for his book ALGEBREN UND IHRE ZAHLENTHEORIE. He served as Editor of the Monthly 1902-1908, and the Transactions from 1911 to 1916, and he was President of the American Mathematical Society from 1916-1918. He was elected to membership in the National Academy of Sciences in 1913 and was a member of the American Philosophical Society, the American Academy of Arts and Sciences, and the London Mathematical Society. He was also a foreign member of the Academy of the Institute of France, and an honorary member of the Czechoslovakian Union of Mathematics and Physics. He was awarded the honorary Sc.D. degree by Harvard in 1936 and Princeton in 1941.

Professor Dickson died in Texas on January 17, 1954.

Dickson was one of our most prolific mathematicians. His bibliography (prepared by $\mathrm{Mr}$. Richard Block, a student at the University of Chicago) contains 285 titles. Of these 18 are books, one a joint book with Miller and Blichfeldt. One of the books is his major threevolume History of the theory of numbers which would be a life's work by itself for a more ordinary man.

Dickson was an inspiring teacher. He supervised the doctorate dissertation of at least 55 Chicago Ph.D's. He helped his students 
to get started in research after the Ph.D. and his books had a worldwide influence in stimulating research.

Attention should be called to the attached bibliography. It includes Dickson's books with titles listed in capitals. It does not include Dickson's portion of the report of the Committee on Algebraic Number Theory, nor does it include Dickson's monograph on ruler and compass constructions which appeared in Monographs on Modern Mathematics.

We now pass on to a brief discussion of Dickson's research.

1. Linear groups. Dickson's first major research effort was a study of finite linear groups. All but seven of his first forty-three papers were on that subject and this portion of his work led to his famous first book, [44]. The linear groups which had been investigated by Galois, Jordan, and Serret were all groups over the field of $p$ elements. Dickson generalized their results to linear groups over an arbitrary finite field. He obtained many new systems of simple groups, and he closed his book with a still valuable summary of the known systems of simple groups.

Dickson's work on linear groups continued until 1908 and he wrote about 44 additional papers on the subject. In these later papers he studied the isomorphism of certain simple groups and questions about the existence of certain types of subgroups. He also derived a number of theorems on infinite linear groups.

2. Finite fields and Chevalley's Theorem. In [44] Dickson gave the first extensive exposition of the theory of finite fields. He applied his deep knowledge of that subject not only to linear groups but to other problems which we shall discuss later. He studied irreducibility questions over a finite field in [113], the Galois theory in [114], and forms whose values are squares in [139]. His knowledge of the role of the non-null form was shown in [155]. In [142] Dickson made the following statement: "For a finite field it seems to be true that every form of degree $m$ in $m+1$ variables vanishes for values not all zero in the field." This result was first proved by C. Chevalley in his paper Démonstration d'une hypothèse de M. Artin, Hamb. Abh. vol. 11 (1935) pp. 73-75. At least the conjecture should have been attributed to Dickson who actually proved the theorem for $m=2,3$.

3. Invariants. Several of Dickson's early papers were concerned with the problems of the algebraic geometry of his time. For example, see [4], [48], [54]. This work led naturally to his study of algebraic invariants and his interest in finite fields to modular invariants. $\mathrm{He}$ wrote a basic paper on the latter subject in [141], and many other papers on the subject. In these papers he devoted a great deal of 
space to the details of a number of special cases. His book, [172], on the classical theory of algebraic invariants, was published in 1914, the year after the appearance of his colloquium lectures. His amazing productivity is attested to by the fact that he also published his book, [173], on linear algebras in 1914.

4. Algebras. Dickson played a major role in research on linear algebras. He began with a study of finite division algebras in [105], [115], [116], and [117]. In these papers he determined all three and four-dimensional finite (non-associative) division algebras over a field of characteristic not two, a set of algebras of dimension six, and a method for constructing algebras of dimension $m k$ with a subfield of dimension $m$. In [126] he related the theory of ternary cubic forms to the theory of three-dimensional division algebras. His last paper on non-associative algebras, [268], appeared in 1937 and contained basic results on algebras of degree two.

Reference has already been made to Dickson's first book on linear algebras. In that text he gave a proof of his result that a real Cayley division algebra is actually a division algebra. He presented the Cartan theory of linear associative algebras rather than the Wedderburn theory but stated the results of the latter theory in his closing chapter without proofs. The present value of this book is enhanced by numerous bibliographical references.

Dickson defined cyclic algebras in a Bulletin abstract of vol. 12 (1905-1906). His paper, [160], on the subject did not appear until 1912 where he presented a study of algebras of dimension 16.

Dickson's work on the arithmetics of algebras first appeared in [204]. His major work on the subject of arithmetics was presented in [213] where he also gave an exposition of the Wedderburn theory. See also [237] and [238].

The text [231] is a German version of [213]. However, the new version also contains the results on crossed product algebras which had been published in [223], and contains many other items of importance.

5. Theory of numbers. Dickson always said that mathematics is the queen of the sciences, and that the theory of numbers is the queen of mathematics. He also stated that he had always wished to work in the theory of numbers and that he wrote his monumental threevolume History of the theory of numbers so that he could know all of the work which had been done in the subject. His first paper, [28], contained a generalization of the elementary Fermat theorem which arose in connection with finite field theory. He was interested in the existence of perfect numbers and wrote [166], and [167] on the re- 
lated topic of abundant numbers. His interest in Fermat's last theorem appears in [190], [136], [137], [138], and [144]. During 1926-1930 he spent most of his energy on research in the arithmetic theory of quadratic forms, in particular on universal forms.

Dickson's interest in additive number theory began in 1927 with [229]. He wrote many papers on the subject during the remainder of his life. The analytic results of Vinogradov gave Dickson the hope of proving the so-called ideal Waring theorem. This he did in a long series of papers. His final result is an almost complete verification of the conjecture made by J. A. Euler in 1772. That conjecture stated that every positive integer is a sum of $J n$th powers where we write $3^{n}=2^{n} q+r, 2^{n}>r>0$, and $J=2^{n}+q-2$. Dickson showed that if $n>6$ this value is correct unless $q+r+3>2^{n}$. It is still not known whether or not this last inequality is possible but if it does occur the number $g(n)$ of such $n$th powers required to represent all integers is $J+f$, or $J+f-1$, according as $f q+f+q=2^{n}$, or $f q+f+q>2^{n}$, where $f$ is the greatest integer in $(4 / 3)^{n}$.

6. Miscellaneous. We close by mentioning Dickson's interest in the theory of matrices which is best illustrated by his text, Modern algebraic theories. His geometric work in [179], [181], [182], [183], [184], [185], and [186] must also be mentioned, as well as his interesting monograph [219] on differential equations from the Lie group standpoint.

\section{BIBLIOGRAPHY}

1. On the inscription of regular polygons, Ann. of Math. vol. 9 (1895) pp. 73-84.

2. Gergonne's pile problem, Bull. Amer. Math. Soc. vol. 1 (1895) pp. 184-186.

3. Cyclic numbers, Quarterly Journal of Pure and Applied Mathematics vol. 27 (1895) pp. 366-377.

4. A quadratic Cremona transformation defined by a conic, Rend. Circ. Mat. Palermo vol. 9 (1895) pp. 256-257.

5. Analytic functions suitable to represent substitutions, Amer. J. Math. vol. 18 (1896) pp. 210-218.

6. The analytic representation of substitutions on a power of a prime number of letters with a discussion of the linear group, Ann. of Math. vol. 11 (1897), Part I, pp. 65-102; Part II, pp. 161-183.

7. A triply infinite system of simple groups, Quarterly Journal of Pure and Applied Mathematics vol. 29 (1897) pp. 169-178.

8. Systems of continuous and discontinuous simple groups, Bull. Amer. Math. Soc. vol. 3 (1897) pp. 265-273.

9. Higher irreducible congruences, Bull. Amer. Math. Soc. vol. 3 (1897) pp. 381389.

10. Orthogonal group in a Galois field, Bull. Amer. Math. Soc. vol. 4 (1898) pp. 196200.

11. Systems of simple groups derived from the orthogonal group, Bull. Amer. Math. Soc. vol. 4 (1898) pp. 382-389. 
12. The structure of the hypoabelian groups, Bull. Amer. Math. Soc. vol. 4 (1898) pp. 495-510.

13. The first hypoabelian group generalized, Quarterly Journal of Pure and Applied Mathematics vol. 30 (1898) pp. 1-16.

14. A new triply-infinite system of simple groups obtained by a twofold generalization of Jordan's first hypoabelian group, Bull. Amer. Math. Soc. vol. 5 (1898) pp. 10-12.

15. Concerning a linear homogeneous group in $C_{m, q}$ variables isomorphic to the general linear homogeneous group in $m$ variables, Bull. Amer. Math. Soc. vol. 5 (1898) pp. 120-135.

16. Simplicity of the Abelian group on two pairs of indices in the Galois field of order $2^{n}, n>1$, Quarterly Journal of Pure and Applied Mathematics vol. 30 (1898) pp. 383384 .

17. The quadratic Cremona transformation, Proceedings of the California Academy of Sciences vol. 1 (1898) pp. 13-23.

18. Systems of simple groups derived from the orthogonal group, Proceedings of the California Academy of Sciences vol. 1 (1898) pp. 29-40.

19. Systems of simple groups derived from the orthogonal groups, Proceedings of the California Academy of Sciences vol. 1 (1899) pp. 47-57.

20. The structure of certain linear groups with quadratic invariants, Proc. London Math. Soc. vol. 30 (1899) pp. 70-98.

21. A class of linear groups including the Abelian one, Quarterly Journal of Pure and Applied Mathematics vol. 31 (1899) pp. 60-66.

22. The group of linear homogeneous substitutions on $m q$ variables which is defined by the invariant $\Phi=\sum_{i=1}^{i=m} \xi_{i 1} \xi_{i 2} \cdots \xi_{i q}$, Proc. London Math. Soc. vol. 30 (1899) pp. 200-208.

23. Determination of the structure of all linear homogeneous groups in a Galois field which are defined by a quadratic invariant, Amer. J. Math. vol. 21 (1899) pp. 193-256.

24. Sur plusieurs groupes linéaires isomorphes au groups simple d'ordre 25920 , C. R. Acad. Sci. Paris vol. 128 (1899) pp. 873-875.

25. The largest linear homogeneous group with an invariant Pfaffian, Bull. Amer. Math. Soc. vol. 5 (1899) pp. 338-342. 475 .

26. The known finite simple groups, Bull. Amer. Math. Soc. vol. 5 (1899) pp. 470-

27. Sur une généralisation du théoréme de Fermat, C. R. Acad. Sci. Paris vol. 128 (1899) pp. 1083-1085.

28. A generalization of Fermat's theorem, Ann. of Math. vol. 1 (1899) pp. 31-36.

29. Report on the recent progress in the theory of linear groups, Bull. Amer. Math. Soc. vol. 6 (1899) pp. 13-27.

30. Concerning the four known simple linear groups of order 25920 , with an introduction to the hyper-abelian linear groups, Proc. London Math. Soc. vol. 31 (1899) pp. $30-68$.

31. The abstract group isomorphic with the symmetric group of $k$ letters, Proc. London Math. Soc. vol. 31 (1899) pp. 351-353.

32. The structure of the linear homogeneous groups defined by the invariant $\lambda_{1} \xi_{1}^{r}$ $+\lambda_{2} \xi_{2}^{r}+\cdots+\lambda_{m} \xi_{m}^{r}$, Math. Ann. vol. 52 (1899) pp. 561-581.

33. Definition of the Abelian, the two hypoabelian, and related linear groups as quotient groups of the groups of isomorphisms of certain elementary groups, Trans. Amer. Math. Soc. vol. 1 (1900) pp. 30-38.

34. Certain subgroups of the Betti-Mathieu group, Amer. J. Math. vol. 22 (1900) pp. 49-54. 
35. A new definition of the general Abelian linear group, Trans. Amer. Math. Soc vol. 1 (1900) pp. 91-96.

36. Canonical form of a linear homogeneous substitution in a Galois field, Amer. J. Math. vol. 22 (1900) pp. 121-137.

37. Proof of the existence of the Galois field of order $p^{r}$ for every integer $r$ and prime number p, Bull. Amer. Math. Soc. vol. 6 (1900) pp. 203-204.

38. Concerning the cyclic subgroups of the simple group $G$ of all linear fractional substitutions of determinant unity in two non-homogeneous variables with coefficients in an arbitrary Galois field, Amer. J. Math. vol. 22 (1900) pp. 231-252.

39. Isomorphism between certain systems of simple linear groups, Bull. Amer. Math Soc. vol. 6 (1900) pp. 323-328.

40. An abstract simple group of order 25920, Proc. London Math. Soc. vol. 32 (1900) pp. 3-16

41. Determination of an abstract simple group of order $2^{7} \cdot 3^{6} \cdot 5 \cdot 7$ holoedrically isomorphic with a certain orthogonal group and with a certain hypoabelian group, Trans. Amer. Math. Soc. vol. 1 (1900) pp. 353-370.

42. Proof of the non-isomorphism of the simple Abelian group on $2 m$ indices and the orthogonal group on $2 m+1$ indices for $m>2$, Quarterly Journal of Pure and Applied Mathematics vol. 32 (1900) pp. 42-63.

43. Linear substitutions commutative with a given substitution, Proc. London Math. Soc. vol. 32 (1900) pp. 165-170.

44. LINEAR GROUPS WITH AN EXPOSITION OF THE GALOIS FIELD THEOR Y, Leipzig, Teubner, 1901, 10+312 pp.

45. Distribution of the ternary linear homogeneous substitutions in a Galois field into complete sets of conjugate substitutions, Amer. J. Math. vol. 23 (1901) pp. 37-40.

46. Canonical forms of quaternary Abelian substitutions in an arbitrary Galois field, Trans. Amer. Math. Soc. vol. 2 (1901) pp. 103-138.

47. Concerning real and complex continuous groups, Bull. Amer. Math. Soc. vol. 7 (1901) pp. 340-350.

48. The configurations of the 27 lines on a cubic surface and the 28 bitangents to a quartic curve, Bull. Amer. Math. Soc. vol. 8 (1901) pp. 63-70.

49. Concerning the Abelian and related linear groups, Proc. London Math. Soc. vol. 33 (1901) pp. 313-325.

50. The alternating group on eight letters and the quaternary linear congruence group modulo two, Math. Ann. vol. 54 (1901) pp. 564-569.

51. Théorie des groupes linéaires dans un domaine arbitraire de rationalité, $\mathrm{C} . \mathrm{R}$. Acad. Sci. Paris, vol. 132 (1901) pp. 1547-1548.

52. Theory of linear groups in an arbitrary field, Trans. Amer. Math. Soc. vol. 2 (1901) pp. 363-394.

53. Representation of linear groups as transitive substitution groups, Amer. J. Math. vol. 23 (1901) pp. 337-377.

54. A class of groups in an arbitrary realm connected with the configuration of the 27 lines on a cubic surface, Quarterly Journal of Pure and Applied Mathematics vol. 33 (1901) pp. 145-173. 192.

55. On systems of isothermal curves, Amer. Math. Monthly vol. 8 (1901) pp. 187-

56. The known systems of simple groups and their inter-isomorphisms, Compte Rendu du Deuxième Congrès International des Mathématiciens, Paris, 1900, Paris, Gauthier-Villars, 1902, pp. 225-229.

57. Cyclic subgroups of the simple ternary linear fractional group in a Galois field, Amer. J. Math. vol. 24 (1902) pp. 1-12. 
58. The groups of Steiner in problems of contact, Trans. Amer. Math. Soc. vol. 3 (1902) pp. 38-45.

59. The groups of Steiner in problems of contact (Second Paper), Trans. Amer. Math. Soc. vol. 3 (1902) pp. 377-382.

60. Canonical form of a linear homogeneous transformation in an arbitrary realm of rationality, Amer. J. Math. vol. 24 (1902) pp. 101-108.

61. Linear groups in an infinite field, Proc. London Math. Soc. vol. 34 (1902) pp. 185-205.

62. Theorems on the residues of multinomial coefficients with respect to a prime modulus, Quarterly Journal of Pure and Applied Mathematics vol. 33 (1902) pp. 378-384.

63. A class of simply transitive linear groups, Bull. Amer. Math. Soc. vol. 8 (1902) pp. 394-401.

64. The hyperorthogonal groups, Math. Ann. vol. 55 (1902) pp. 521-572.

65. An elementary exposition of Frobenius's theory of group-characters and groupdeterminants, Ann. of Math. vol. 4 (1902) pp. 25-49.

66. On the group defined for any given field by the multiplication table of any given finite group, Trans. Amer. Math. Soc. vol. 3 (1902) pp. 285-301.

67. A matrix defined by the quaternion group, Amer. Math. Monthly vol. 9 (1902) pp. 243-248.

68. COLLEGE ALGEBRA, New York, Wiley, 1902, $7+214$ pp.

69. Ternary orthogonal group in a general field, University of Chicago Press, 1903, 8 pp.

70. Groups defined for a general field by the rotation groups, University of Chicago Press, 1903, 17 pp.

71. INTRODUCTION TO THE THEORY OF ALGEBRAIC EQUATIONS, New York, Wiley, 1903, 5+104 pp.

72. Definitions of a field by independent postulates, Trans. Amer. Math. Soc. vol. 4 (1903) pp. 13-20.

73. Definitions of a linear associative algebra by independent postulates, Trans. Amer. Math. Soc. vol. 4 (1903) pp. 21-26.

74. On the groups defined for an arbitrary field by the multiplication tables of certain finite groups, Proc. London Math. Soc. vol. 35 (1903) pp. 68-80.

75. Three sets of generational relations defining the abstract simple group of order 504, Bull. Amer. Math. Soc. vol. 9 (1903) pp. 194-204.

76. Generational relations defining the abstract simple group of order 660 , Bull. Amer. Math. Soc. vol. 9 (1903) pp. 204-206.

77. The abstract group $G$ simply isomorphic with the alternating group on six letters, Bull. Amer. Math. Soc. vol. 9 (1903) pp. 303-306.

78. Fields whose elements are linear differential expressions, Bull. Amer. Math. Soc. vol. 10 (1903) pp. 30-31.

79. On the subgroups of order a power of $p$ in the quaternary Abelian group in the Galois field of order $p^{n}$, Trans. Amer. Math. Soc. vol. 4 (1903) pp. 371-386.

80. On the reducibility of linear groups, Trans. Amer. Math. Soc. vol. 4 (1903) pp. $434-436$.

81. The abstract group simply isomorphic with the group of linear fractional transformations in a Galois field, Proc. London Math. Soc. vol. 35 (1903) pp. 292-305.

82. Generational relations of an abstract simple group of order 4080, Proc. London Math. Soc. vol. 35 (1903) pp. 306-319.

83. Generational relations for the abstract group simply isomorphic with the linear fractional group in the $G F\left[2^{n}\right]$, Proc. London Math. Soc. vol. 35 (1903) pp. 443-454. 
84. 1. Ternary orthogonal groups in a general field. 2. The groups defined for a general field by the rotation group, University of Chicago Press, 1904, $26 \mathrm{pp}$.

85. The subgroups of order a power of 2 of the simple quinary orthogonal group in the Galois field of order $p^{n}=8 l \pm 3$, Trans. Amer. Math. Soc. vol. 5 (1904) pp. 1-38.

86. Determination of all groups of binary linear substitutions with integral coefficients taken modulo 3 and of determinant unity, Ann. of Math. vol. 5 (1904) pp. 140-144.

87. Determination of all subgroups of the known simple group of order 25920, Trans. Amer. Math. Soc. vol. 5 (1904) pp. 126-166.

88. Two systems of subgroups of the quaternary Abelian group in a general Galois field, Bull. Amer. Math. Soc. vol. 10 (1904) pp. 178-184.

89. A new extension of Dirichlet's theorem on prime numbers, Messenger of Mathematics, vol. 33 (1904) pp. 155-161.

90. Addition to the paper on the four known simple linear groups of order 25920, Proc. London Math. Soc. vol. 1 (1904) pp. 283-284.

91. On the subgroups of order a power of $p$ in the linear homogeneous and fractional groups in the GF[ $\left.p^{n}\right]$, Bull. Amer. Math. Soc. vol. 10 (1904) pp. 385-397.

92. Memoir on Abelian transformations, Amer. J. Math. vol. 26 (1904) pp. 243-318.

93. Application of groups to a complex problem in arrangements, Ann. of Math. vol. 6 (1904) pp. 31-34.

94. On the minimum degree of resolvants for the p-section of the periods of hyperelliptic functions of four periods, Jber. Deutschen Math. Verein. vol. 13 (1904) pp. 559-560.

95. A property of the group $G_{2}^{2 n}$ all of whose operators except identity are of period 2, Amer. Math. Monthly vol. 11 (1904) pp. 203-206.

96. The groups of a tactical configuration, Bull. Amer. Math. Soc. vol. 11 (1905) pp. 177-179.

97. The minimum degree $\tau$ of resolvents for the p-section of the periods of hyperelliptic functions of four periods, Trans. Amer. Math. Soc. vol. 6 (1905) pp. 48-57.

98. On the cyclotomic functions, Amer. Math. Monthly vol. 12 (1905) pp. 86-89.

99. On the real elements of certain classes of geometrical configurations, Ann. of Math. vol. 6 (1905) pp. 141-150.

100. A new system of simple groups, Math. Ann. vol. 60 (1905) pp. 137-150.

101. Determination of the ternary modular groups, Amer. J. Math. vol. 27 (1905) pp. 189-202.

102. Definitions of a group and a field by independent postulates, Trans. Amer. Math. Soc. vol. 6 (1905) pp. 198-204.

103. On semi-groups and the general isomorphism between finite groups, Trans. Amer. Math. Soc. vol. 6 (1905) pp. 205-208.

104. Determination of all the subgroups of the three highest powers of $p$ in the group $G$ of all m-ary linear homogeneous transformations modulo $p$, Quarterly Journal of Pure and Applied Mathematics vol. 36 (1905) pp. 373-384.

105. On finite algebras, Nachr. Ges. Wiss. Göttingen (1905) pp. 358-393.

106. On the class of the substitutions of various linear groups, Bull. Amer. Math. Soc. vol. 11 (1905) pp. 426-432.

107. Subgroups of order a power of $p$, in the general and special m-ary linear homogeneous groups in the GF[ $\left.p^{n}\right]$, Amer. J. Math. vol. 27 (1905) pp. 280-302.

108. A general theorem on algebraic numbers, Bull. Amer. Math. Soc. vol. 11 (1905) pp. $482-486$.

109. On hypercomplex number systems, Trans. Amer. Math. Soc. vol. 6 (1905) pp. 344-348. 
110. Expressions for the elements of a determinant of an array, Amer. Math. Monthly vol. 12 (1905) pp. 217-221.

111. On the quaternary linear homogeneous groups modulo $p$ of order a multiple of $p$, Amer. J. Math. vol. 28 (1906) pp. 1-16.

112. On quadratic, hermitian and bilinear forms, Trans. Amer. Math. Soc. vol. 7 (1906) pp. 275-292.

113. Criteria for the irreducibility of functions in a finite field, Bull. Amer. Math. Soc. vol. 13 (1906) pp. 1-8.

114. On the theory of equations in a Galois field, Bull. Amer. Math. Soc. vol. 13 (1906) pp. 8-10.

115. Linear algebras in which division is always uniquely possible, Trans. Amer. Math. Soc. vol. 7 (1906) pp. 370-390.

116. On linear algebras, Amer. Math. Monthly vol. 13 (1906) pp. 201-205.

117. On commutative linear algebras in which division is always uniquely possible, Trans. Amer. Math. Soc. vol. 7 (1906) pp. 514-522.

118. The abstract form of the special linear homogeneous group in an arbitrary field, Quarterly Journal of Pure and Applied Mathematics vol. 38 (1907) pp. 141-145.

119. The abstract form of the Abelian linear groups, Quarterly Journal of Pure and Applied Mathematics vol. 38 (1907) pp. 145-158.

120. The symmetric group on eight letters and the senary first hypoabelian group, Bull. Amer. Math. Soc. vol. 13 (1907) pp. 386-389.

121. Modular theory of group characters, Bull. Amer. Math. Soc. vol. 13 (1907) pp. 477-488.

122. Invariants of binary forms under modular transformations, Trans. Amer. Math. Soc. vol. 8 (1907) pp. 205-232.

123. Invariants of the general quadratic form modulo 2, Proc. London Math. Soc. vol. 5 (1907) pp. 301-324.

124. Modular theory of group-matrices, Trans. Amer. Math. Soc. vol. 8 (1907) pp. 389-398.

125. On quadratic forms in a general field, Bull. Amer. Math. Soc. vol. 14 (1907) pp. $108-115$.

126. On triple algebras and ternary cubic forms, Bull. Amer. Math. Soc. vol. 14 (1908) pp. 160-169.

127. The Galois group of a reciprocal quartic equation, Amer. Math. Monthly vol. 15 (1908) pp. 71-78.

128. A class of groups in an arbitrary realm connected with the configuration of the 27 lines on a cubic surface. Second paper, Quarterly Journal of Pure and Applied Mathematics vol. 39 (1908) pp. 205-209.

129. On the canonical forms and automorphs of ternary cubic forms, Amer. J. Math. vol. 30 (1908) pp. 117-128.

130. Representations of the general symmetric group as linear groups in finite and infinite fields, Trans. Amer. Math. Soc. vol. 9 (1908) pp. 121-148.

131. On families of quadratic forms in a general field, Quarterly Journal of Pure and Applied Mathematics vol. 39 (1908) pp. 316-333.

132. On higher congruences and modular invariants, Bull. Amer. Math. Soc. vol. 14 (1908) pp. 313-318.

133. On the factorization of large numbers, Amer. Math. Monthly vol. 15 (1908) pp. 217-222.

134. Criteria for the irreducibility of a reciprocal equation, Bull. Amer. Math. Soc. vol. 14 (1908) pp. 426-430. 
135. Invariantive reduction of quadratic forms in the GF [2n], Amer. J. Math. vol. 30 (1908) pp. 263-281.

136. On the last theorem of Fermat, Messenger of Mathematics vol. 38 (1908) pp. $14-32$

137. On the last theorem of Fermat, II, Quarterly Journal of Pure and Applied Mathematics vol. 40 (1908) pp. 27-45.

138. On the congruence $x^{n}+y^{n}+z^{n} \equiv 0(\bmod p)$, J. Reine Angew. Math. vol. 135 (1908) pp. 134-141.

139. Definite forms in a finite field, Trans. Amer. Math. Soc. vol. 10 (1909) pp. 109122.

140. Rational reduction of a pair of binary quadratic forms; their modular invariants, Amer. J. Math. vol. 31 (1909) pp. 103-146.

141. General theory of modular invariants, Trans. Amer. Math. Soc. vol. 10 (1909) pp. 123-158.

142. On the representation of numbers by modular forms, Bull. Amer. Math. Soc. vol. 15 (1909) pp. 338-347.

143. (with M. Kaba), On the representation of numbers as the sum of two squares, Amer. Math. Monthly vol. 16 (1909) pp. 85-87.

144. Lower limit for the number of sets of solutions of $x^{b}+y^{a}+z^{e} \equiv 0$ (mod. $p$ ), J. Reine Angew. Math. vol. 135 (1909) pp. 181-188.

145. On commutative linear groups, Quarterly Journal of Pure and Applied Mathematics vol. 40 (1909) pp. 167-196.

146. A theory of invariants, Amer. J. Math. vol. 31 (1909) pp. 337-354.

147. Equivalence of pairs of bilinear or quadratic forms under rational transformation, Trans. Amer. Math. Soc. vol. 10 (1909) pp. 347-360.

148. Combinants, Quarterly Journal of Pure and Applied Mathematics vol. 40 (1909) pp. 349-365.

149. On certain diophantine equations, Messenger of Mathematics vol. 39 (1909) pp. 86-87.

150. Modular invariants of a general system of linear forms, Proc. London Math. Soc. vol. 7 (1909) pp. 430-444.

151. On the factorization of integral functions with p-adic coefficients, Bull. Amer. Math. Soc. vol. 17 (1910) pp. 19-23.

152. An invariantive investigation of irreducible binary modular forms, Trans. Amer. Math. Soc. vol. 12 (1911) pp. 1-18.

153. A fundamental system of the general modular linear group with a solution of the form problem, Trans. Amer. Math. Soc. vol. 12 (1911) pp. 75-98.

154. Note on modular invariants, Quarterly Journal of Pure and Applied Mathematics vol. 42 (1911) pp. 158-161.

155. On non-vanishing forms, Quarterly Journal of Pure and Applied Mathematics vol. 42 (1911) pp. 162-171.

156. Binary modular groups and their invariants, Amer. J. Math. vol. 33 (1911) pp. 175-192.

157. Notes on the theory of numbers, Amer. Math. Monthly vol. 18 (1911) pp. 109110.

158. On the negative discriminants for which there is a single class of positive binary quadratic forms, Bull. Amer. Math. Soc. vol. 17 (1911) pp. 534-547.

159. Note on cubic equations and congruences, Ann. of Math. vol. 12 (1911) pp. 149-152.

160. Linear algebras, Trans. Amer. Math. Soc. vol. 13 (1912) pp. 59-73.

161. Amicable number triples, Amer. Math. Monthly vol. 20 (1913) pp. 84-92. 
162. On the rank of a symmetrical matrix, Ann. of Math. vol. 15 (1913) pp. 27-28.

163. Theorems and tables on the sum of the divisors of a number, Quarterly Journal of Pure and Applied Mathematics vol. 44 (1913) pp. 264-296.

164. Proof of the finiteness of the modular covariants, Trans. Amer. Math. Soc. vol. 14 (1913) pp. 299-310.

165. On binary modular groups and their invariants, Bull. Amer. Math. Soc. vol. 20 (1913) pp. 132-134.

166. Finiteness of the odd perfect and primitive abundant numbers with $n$ distinct prime factors, Amer. J. Math. vol. 35 (1913) pp. 413-422.

167. Even abundant numbers, Amer. J. Math. vol. 35 (1913) pp. 423-426.

168. The invariants, seminvariants and linear covariants of the binary quartic form modulo 2, Ann. of Math. vol. 15 (1914) pp. 114-117.

169. Linear associative algebras and Abelian equations, Trans. Amer. Math. Soc. vol. 15 (1914) pp. 31-46.

170. ON INVARIANTS AND THE THEORY OF NUMBERS, Amer. Math. Soc. Colloquium Publications, vol. 4, 1914, pp. 1-110.

171. ELEMENTARY THEORY OF EQUATIONS, New York, Wiley, 1914, $5+184 \mathrm{pp}$.

172. ALGEBRAIC INVARIANTS, New York, Wiley, 1914, 10+100 pp. [Mathematical monographs, edited by M. Merriman and R. S. Woodward, No. 14.]

173. LINEAR ALGEBRAS, Cambridge University Press, $1914,8+73$ pp. [Cambridge Tracts in Math. and Math. Physics, No. 16.]

174. The points of inflection of a plane cubic curve, Ann. of Math. (2) vol. 16 (1914) pp. 50-66.

175. Invariants in the theory of numbers, Trans. Amer. Math. Soc. vol. 15 (1914) pp. 497-503.

176. On the trisection of an angle and the construction of regular polygons of 7 and 9 sides, Amer. Math. Monthly vol. 21 (1914) pp. 259-262.

177. Modular invariants of the system of a binary cubic, quadratic and linear form, Quarterly Journal of Pure and Applied Mathematics vol. 45 (1914) pp. 373-384.

178. Recent progress in the theories of modular and formal invariants and in modular geometry, Proc. Nat. Acad. Sci. U.S.A. vol. 1 (1915) pp. 1-4.

179. Projective classification of cubic surfaces modulo 2, Ann. of Math. (2) vol. 16 (1915) pp. 139-157.

180. Invariants, seminvariants, and covariants of the ternary and quaternary quadratic form modulo 2, Bull. Amer. Math. Soc. vol. 21 (1915) pp. 174-179.

181. Invariantive theory of plane cubic curves modulo 2, Amer. J. Math. vol. 37 (1915) pp. 107-116.

182. Quartic curves modulo 2, Trans. Amer. Math. Soc. vol. 16 (1915) pp. 111-120.

183. Classification of quartic curves, modulo 2, Messenger of Mathematics vol. 44 (1915) pp. 189-192.

184. The straight lines on modular cubic surfaces, Proc. Nat. Acad. Sci. U.S.A. vol. 1 (1915) pp. 248-253.

185. Geometrical and invariantive theory of quartic curves modulo 2, Amer. J. Math. vol. 37 (1915) pp. 337-354.

186. Invariantive classification of pairs of conics modulo 2, Amer. J. Math. vol. 37 (1915) pp. 355-358.

187. On the relation between linear algebras and continuous groups, Bull. Amer. Math. Soc. vol. 22 (1915) pp. 53-61.

188. (with G. A. Miller and H. F. Blichfeldt), THEORY AND APPLICATIONS OF FINITE GROUPS, New York, Wiley, 1916, 17+390 pp. 
189. An extension of the theory of numbers by means of correspondences between fields, Bull. Amer. Math. Soc. vol. 23 (1916) pp. 109-111.

190. Fermat's last theorem and the origin and nature of the theory of algebraic numbers, Ann. of Math. (2) vol. 18 (1917) pp. 161-187.

191. On quaternions and their generalization and the history of the eight square theorem, Ann. of Math. (2) vol. 20 (1919) pp. 155-171, 297.

192. Mathematics in war perspective, Bull. Amer. Math. Soc. vol. 25 (1919) pp. 289-311.

193. Applications of the geometry of numbers to algebraic numbers, Bull. Amer. Math. Soc. vol. 25 (1919) pp. 453-455.

194. HISTORY OF THE THEORY OF NUMBERS, vol. I, DIVISIBILITY AND PRIMALITY, Carnegie Institution of Washington, Publication No. 256, $1919,12+486 \mathrm{pp}$.

195. HISTORY OF THE THEORY OF NUMBERS, Vol. II. DIOPHA NTINE ANAL YSIS, Carnegie Institution of Washington, Publication No. 256, 1920, $12+803$ pp.

196. Les polynomes egaux a des determinants, C. R. Acad. Sci. Paris vol. 171 (1920) pp. 1360-1362.

197. Some relations between the theory of numbers and other branches of mathematics, Conférence générale, Comptes Rendus du Congrés International des Mathématiciens, Strasbourg, 1920, Toulouse, H. Villat, 1921, pp. 41-56.

198. Homogeneous polynomials with a multiplication theorem, Comptes Rendus du Congrés International des Mathématiciens, Strasbourg, 1920, Toulouse, H. Villat, 1921, pp. 215-230.

199. Algebraic theory of the expressibility of cubic forms as determinants, with application to diophantine analysis, Amer. J. Math. vol. 43 (1921) pp. 102-125.

200. Quaternions and their generalizations, Proc. Nat. Acad. Sci. U.S.A. vol. 7 (1921) pp. 109-114.

201. Fallacies and misconceptions in diophantine analysis, Bull. Amer. Math. Soc. vol. 27 (1921) pp. 312-319.

202. Determination of all general homogeneous polynomials expressible as determinants with linear elements, Trans. Amer. Math. Soc. vol. 22 (1921) pp. 167-179.

203. A new method in diophantine analysis, Bull. Amer. Math. Soc. vol. 27 (1921) pp. 353-365. 232.

204. Arithmetic of quaternions, Proc. London Math. Soc. vol. 20 (1921) pp. 225-

205. Rational triangles and quadrilaterals, Amer. Math. Monthly vol. 28 (1921) pp. 244-250.

206. Reducible cubic forms expressible rationally as determinants, Ann. of Math. vol. 23 (1921) pp. 70-74.

207. A fundamental system of covariants of the ternary cubic form, Ann. of Math. vol. 23 (1921) pp. 78-82.

208. La composition des polynomes, C. R. Acad. Sci. Paris vol. 172 (1921) pp. 636-640.

209. PLANE TRIGONOMETRY WITH PRACTICAL APPLICATIONS, Chicago, Sanborn, $1922,12+176+35$ pp.

210. A FIRST COURSE IN THE THEORY OF EQUATIONS, New York, Wiley, $1922,6+168 \mathrm{pp}$.

211. Impossibility of restoring unique factorization in a hypercomplex arithmetic, Bull. Amer. Math. Soc. vol. 28 (1922) pp. 438-442. 
212. The rational linear algebras of maximum and minimum ranks, Proc. London Math. Soc. (2) vol. 22 (1923) pp. 143-162.

213. ALGEBRAS AND THEIR ARITHMETICS, Chicago, University of Chicago Press, $1923,12+241 \mathrm{pp}$.

214. HISTORY OF THE THEORY OF NUMBERS, Vol. III. QUADRATIC AND HIGHER FORMS (With a chapter on the class number by G. H. Cresse), Carnegie Institution of Washington, Publication No. 256, 1923, 4+313 pp.

215. A new simple theory of hypercomplex integers, J. Math. Pures Appl. (9) vol. 2 (1923) pp. 281-326.

216. Integral solutions of $x^{2}-m y^{2}=z w$, Bull. Amer. Math. Soc. vol. 29 (1923) pp. 464-467.

217. On the theory of numbers and generalized quaternions, Amer. J. Math. vol. 46 (1924) pp. 1-16.

218. Algebras and their arithmetics, Bull. Amer. Math. Soc. vol. 30 (1924) pp. 247-257.

219. Differential equations from the group standpoint, Ann. of Math. (2) vol. 25 (1924) pp. 287-378.

220. Quadratic fields in which factorization is always unique, Bull. Amer. Math. Soc. vol. 30 (1924) pp. 328-334. 838.

221. Algebres nouvelles de division, C. R. Acad. Sci. Paris vol. 181 (1925) pp. 836-

222. Resolvent sextics of quintic equations, Bull. Amer. Math. Soc. vol. 31 (1925) pp. 515-523.

223. New division algebras, Trans. Amer. Math. Soc. vol. 28 (1926) pp. 207-234. 224. MODERN ALGEBRAIC THEORIES, Chicago, New York, Boston; Sanborn, 1926, $9+276 \mathrm{pp}$.

225. All integral solutions of $a x^{2}+b x y+c y^{2}=w_{1} w_{2} \cdots w_{n}$, Bull. Amer. Math. Soc. vol. 32 (1926) pp. 644-648.

226. Quadratic forms which represent all integers, Proc. Nat. Acad. Sci. U.S.A. vol. 12 (1926) pp. 756-757.

227. Quaternary quadratic forms representing all integers, Amer. J. Math. vol. 49 (1927) pp. 39-56.

228. Integers represented by positive ternary quadratic forms, Bull. Amer. Math. Soc. vol. 33 (1927) pp. 63-70.

229. Extensions of Waring's theorem on nine cubes, Amer. Math. Monthly vol. 34 (1927) pp. 177-183.

230. A generalization of Waring's theorem on nine cubes, Bull. Amer. Math. Soc. vol. 33 (1927) pp. 299-300.

231. ALGEBREN UND IHRE ZAHLENTHEORIE, Zurich, Orell Füssli, 1927, $8+308 \mathrm{pp}$. (translation of completely revised and extended manuscript).

232. Extensions of Waring's theorem on fourth powers, Bull. Amer. Math. Soc. vol. 33 (1927) pp. 319-327.

233. Singular case of pairs of bilinear, quadratic, or Hermitian forms, Trans. Amer. Math. Soc. vol. 29 (1927) pp. 239-253.

234. Generalization of Waring's theorem on fourth, sixth, and eighth powers, Amer. J. Math. vol. 49 (1927) pp. 241-250.

235. Ternary quadratic forms and congruences, Ann. of Math. (2) vol. 28 (1927) pp. 333-341.

236. All positive integers are sums of values of a quadratic function of $x$, Bull. Amer. Math. Soc. vol. 33 (1927) pp. 713-720. 
237. Outline of the theory to date of the arithmetics of algebras, Proceedings of the International Mathematical Congress held in Toronto, 1924, Toronto, The University of Toronto Press, 1928, vol. 1, pp. 95-102.

238. Further development of the theory of arithmetics of algebras, Proceedings of the International Mathematical Congress held in Toronto, 1924, Toronto, The University of Toronto Press, 1928, vol. 1, pp. 173-184.

239. A new theory of linear transformations and pairs of bilinear forms, Proceedings of the International Mathematical Congress, held in Toronto, 1924, Toronto, The University of Toronto Press, 1928, vol. 1, pp. 361-363.

240. Simpler proofs of Waring's theorem on cubes with various generalizations, Trans. Amer. Math. Soc. vol. 30 (1928) pp. 1-18.

241. Additive number theory for all quadratic functions, Amer. J. Math. vol. 50 (1928) pp. 1-48.

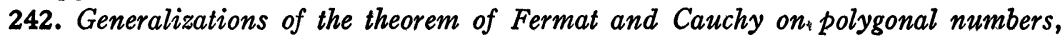
Bull. Amer. Math. Soc. vol. 34 (1928) pp. 63-72.

243. Extended polygonal numbers, Bull. Amer. Math. Soc. vol. 34 (1928) pp. 205217.

244. Quadratic functions or forms, sums of whose values give all positive integers, J. Math. Pures Appl. vol. 7 (1928) pp. 319-336.

245. New division algebras, Bull. Amer. Math. Soc. vol. 34 (1928) pp. 555-560.

246. Universal quadratic forms, Trans. Amer. Math. Soc. vol. 31 (1929) pp. 164189.

247. The forms $a x^{2}+b y^{2}+c z^{2}$ which represent all integers, Bull. Amer. Math. Soc. vol. 35 (1929) pp. 55-59.

248. INTRODUCTION TO THE THEORY OF NUMBERS, Chicago, The University of Chicago Press, $1929,8+183$ pp.

249. Construction of division algebras, Trans. Amer. Math. Soc. vol. 32 (1930) pp. 319-334.

250. STUDIES IN THE THEORY OF NUMBERS, Chicago, The University of Chicago Press (The University of Chicago Science Series), 1930, 10+230 pp.

251. Proof of a Waring theorem on fifth powers, Bull. Amer. Math. Soc. vol. 37 (1931) pp. 549-553.

252. MINIMUM DECOMPOSITIONS INTO FIFTH POWERS, Vol. III, Mathematical Tables, British Assoc. for the Advancement of Science (Committee for the Calculation of Mathematical Tables), London, Office of the British Assoc., $1933,368 \mathrm{pp}$.

253. Eliakim Hastings Moore, Science (2) vol. 77 (1933) pp. 79-80.

254. Minimum decompositions into n-th powers, Amer. J. Math. vol. 55 (1933) pp. 593-602.

255. Recent progress on Waring's theorem and its generalizations, Bull. Amer. Math. Soc. vol. 39 (1933) pp. 701-727.

256. Waring's problem for cubic functions, Trans. Amer. Math. Soc. vol. 36 (1934) pp. 1-12.

257. Waring's problem for ninth powers, Bull. Amer. Math. Soc. vol. 40 (1934) pp. $487-493$.

258. Universal Waring theorem for eleventh powers, J. London Math. Soc. vol. 9 (1934) pp. 201-206.

259. Two-fold generalizations of Cauchy's lemma, Amer. J. Math. vol. 56 (1934) pp. $513-528$.

260. The converse of Waring's problem, Bull. Amer. Math. Soc. vol. 40 (1934) pp. 711-714. 
261. A new method for universal Waring theorems with details for seventh powers. Amer. Math. Monthly vol. 41 (1934) pp. 547-555.

262. A new method for Waring theorems with polynomial summands, Trans. Amer. Math. Soc. vol. 36 (1934) pp. 731-748.

263. Polygonal numbers and related Waring problems, Quart. J. Math. Oxford Ser. vol. 5 (1934) pp. 283-290.

264. Congruences involving only e-th powers, Acta Arithmetica vol. 1 (1935) pp. 161-167.

265. Cyclotomy and trinomial congruences, Trans. Amer. Math. Soc. vol. 37 (1935) pp. 363-380.

266. Cyclotomy, higher congruences, and Waring's problem, Amer. J. Math. vol. 57 (1935) pp. 391-424.

267. Cyclotomy, higher congruences, and Waring's problem, II, Amer. J. Math. vol. 57 (1935) pp. 463-474.

268. Linear algebras with associativity not assumed, Duke Math. J. vol. 1 (1935) pp. 113-125.

269. Cyclotomy when e is composite, Trans. Amer. Math. Soc. vol. 38 (1935) pp. 187-200.

270. Universal Waring theorems with cubic summands, Acta Arithmetica vol. 1 (1935) pp. 184-196 and Prace Matematyczno-Fizyczne vol. 43 (1936) pp. 223-235.

271. Waring theorems of new type, Amer. J. Math. vol. 58 (1936) pp. 241-248.

272. On Waring's problem and its generalization, Ann. of Math. (2) vol. 37 (1936) pp. 293-316.

273. A new method for Waring theorems with polynomial summands. II, Trans. Amer. Math. Soc. vol. 39 (1936) pp. 205-208.

274. Universal Waring theorems, Mh. Math. Phys. vol. 43 (1936) pp. 391-400.

275. The ideal Waring theorem for twelfth powers, Duke Math. J. vol. 2 (1936) pp. 192-204.

276. Proof of the ideal Waring theorem for exponents 7-180, Amer. J. Math. vol. 58 (1936) pp. 521-529.

277. Solution of Waring's problem, Amer. J. Math. vol. 58 (1936) pp. 530-535.

278. A generalization of Waring's problem, Bull. Amer. Math. Soc. vol. 42 (1936) pp. 525-529.

279. The Waring problem and its generalizations, Bull. Amer. Math. Soc. vol. 42 (1936) pp. 833-842.

280. New Waring theorems for polygonal numbers, Quart. J. Math. Oxford Ser. vol. 8 (1937) pp. 62-65.

281. Universal forms $\sum a_{i} x_{i}^{n}$ and Waring's problem, Acta Arithmetica vol. 2 (1937) pp. 177-196.

282. NEW FIRST COURSE IN THE THEORY OF EQUATIONS, New York, Wiley, $1939,9+185 \mathrm{pp}$.

283. All integers except 23 and 239 are sums of eight cubes, Bull. Amer. Math. Soc. vol. 45 (1939) pp. 588-591.

284. MODERN ELEMENTARY THEORY OF NUMBERS, University of Chicago Press, 1939, $7+309$ pp.

285. Obituary: Hans Frederik Blichfeldt, 1873-1945, Bull. Amer. Math. Soc. vol. 53 (1947) pp. 882-883. 